Pacific Journal of Mathematics

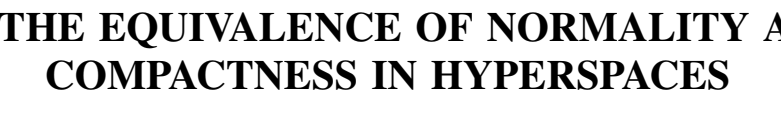




\title{
ON THE EQUIVALENCE OF NORMALITY AND COMPACTNESS IN HYPERSPACES
}

\section{JAMES KEESLING}

\begin{abstract}
Let $X$ be a topological space and $2^{X}$ the space of all closed subsets of $X$ with the finite topology. Assuming the continuum hypothesis it is shown that $2^{X}$ is normal if and only if $X$ is compact. It is not known if the continuum hypothesis is a necessary assumption, but it is shown that for $X$ a $k$-space, $2^{X}$ normal implies $X$ compact. A theorem about the compactification of the $n$-th symmetric product of a space $X$ is first proved which then plays an important part in the proof of the above results.
\end{abstract}

Throughout this paper we will assume that $X$ is any completely regular $T_{1}$ space. By $2^{X}$ we will mean the space of all closed subsets of $X$ with the finite topology [13, Definition 1.7, p. 153] except that we include the empty set as an isolated point as in [12]. The finite topology is also known as the exponential or Vietoris topology. Let $\mathscr{F}_{n}(X)$ be the subspace of $2^{X}$ consisting of all nonempty subsets of $X$ with $n$ points or less. This space is known as the $n$-th symmetric product of $X$.

In this paper the normality of $2^{X}$ is studied. If $X$ is compact, it is known that $2^{x}$ is compact Hausdorff [13, Th. 4.2, p. 161] and thus normal. The main result of this paper is that if we assume the continuum hypothesis $(\mathrm{CH})$, then $2^{X}$ is normal if and only if $X$ is compact. The first result in this direction was obtained by Ivanova [9] who proved that if $X$ is a well ordered space with the order topology, then $2^{X}$ normal implies $X$ compact. In [10] it is shown that $2^{2^{X}}$ is normal if and only if $X$ is compact. These results were obtained without the use of $\mathrm{CH}$.

The paper is divided into three sections. In the first section our main result is that $\mathscr{F}_{n}(\beta X)=\beta \mathscr{F}_{n}(X)$ if and only if $\mathscr{F}_{n}(X)$ is pseudocompact. This result is related to the work of Glicksberg in [7] and the proof makes use of his work. In the second section of the paper we investigate the normality of $2^{X}$ without the aid of $\mathrm{CH}$ using the results of the first section. One significant result in this section is that if $2^{X}$ is normal with $X$ noncompact, then $X$ is normal and countably compact, but $X^{n}$ is not pseudocompact for some $n$. As a corollary one obtains that if $X$ is first countable or locally compact, then $2^{X}$ normal implies that $X$ is compact. Also $2^{X} \times \beta N$ is normal only when $X$ is compact.

In the last section of the paper it is shown that if $\mathrm{CH}$ is assumed, 
then if $2^{X}$ is normal, then $X$ is compact. This result is related to a result of N. Noble [15] who has shown that if every power $X^{\alpha}$ of $X$ is normal, then $X$ is compact. Noble's result does not require $\mathrm{CH}$, however.

Preliminaries. As remarked in the introduction we assume that $X$ is completely regular and $T_{1}$. We denote the Stone-Čech compactification of $X$ by $\beta X$. One can imbed the space $\mathscr{F}_{n}(X)$ into the space $\mathscr{F}_{n}(\beta X)$ by the map $i(F)=F$ for all $F \in \mathscr{F}_{n}(X)$. This imbedding can be easily seen to be onto a dense subset of $\mathscr{F}_{n}(\beta X)$. Since $\mathscr{F}_{n}(\beta X)$ is compact, we thus have a compactification of $\mathscr{F}_{n}(X)$ by $\mathscr{F}_{n}(\beta X)$. By $\beta \mathscr{F}_{n}(X)=\mathscr{F}_{n}(\beta X)$ we mean that this compactification is equivalent to the Stone-Čech compactification of $\mathscr{F}_{n}(X)$.

General background in hyperspaces is conveniently given in [12] and [13]. Use is also made of techniques and results in [10]. Let us recall at this point two results to be used subsequently in the paper. If $K$ is a closed subset of $X$, then $2^{K}$ as a topological space has the same topology as $2^{K}$ has as a subspace of $2^{X}$. If $X=K_{1} \cup K_{2}$ with $K_{1}$ and $K_{2}$ disjoint closed sets, then $2^{X}$ is equivalent to $2^{K_{1}} \times 2^{K_{2}}$ by [12, Corollary 5(a), p. 166].

We consider the cardinals as a subset of the ordinals in the natural way. Infinite cardinals will be denoted by $\omega_{\alpha}$ where $\alpha$ is an ordinal and where $\omega_{0}$ is the cardinality of the integers, $\omega_{1}$ the first uncountable ordinal, etc. $\mathrm{By} \mathrm{CH}$ is meant $2^{\omega_{0}}=\omega_{1}$. This assumption is made only in the last section of the paper.

1. On the compactification of $\mathscr{F}_{n}(X)$. In this section we establish the result $\beta \mathscr{F}_{n}(X)=\mathscr{F}_{n}(\beta X)$ if and only if $\mathscr{F}_{n}(X)$ is pseudocompact. We first show that $\mathscr{F}_{n}(X)$ is pseudocompact if and only if $X^{n}$ is. Our proof of this result is not the easiest possible; however, by establishing an important proposition at this time, the proof of our main result in this section is made easier.

Proposition 1.1. If $X^{n}$ is not pseudocompact, then there is a collection of nonempty open sets in $X\left\{U_{k}^{i}: k=1, \cdots, n ; i=1,2, \cdots\right\}$ such that $\bar{U}_{k}^{i} \cap \bar{U}_{h}^{j}=\phi$ for $(i, k) \neq(j, h)$ and such that if

$$
O_{i}=U_{1}^{i} \times \cdots \times U_{n}^{i}
$$

then $\left\{O_{i}\right\}_{i=1}^{\infty}$ forms a discrete collection in $X^{n}$.

One should compare Proposition 1.1 with that in Isbell [8, 38, p. 139] for motivation. We will prove the following lemma before proving 1.1 .

Lemma 1.2. Suppose that $X^{n}$ has a countable closed discrete 
subset $B=\left\{x^{i}\right\}_{i=1}^{\infty}$ such that (1) $x^{i} \in U_{i}$ with $U_{i}$ open in $X^{n}$; (2) for each subsequence $B^{\prime}=\left\{x^{i}\right\}_{j=1}^{\infty}$ of $B$ and each projection $\pi_{k}, \mathrm{C1}_{X} \pi_{k}\left[B^{\prime}\right]$ is not compact; and (3) for each $i, x^{i}=\left(x_{1}^{i}, \cdots, x_{n}^{i}\right)$ with $x_{j}^{i} \neq x_{k}^{i}$ for $j \neq k$. Then there is a subsequence $B^{\prime}=\left\{x^{i j}\right\}_{j=1}^{\infty}$ of $B$ and a collection of open sets in $X,\left\{V_{k}^{j}: k=1, \cdots, n ; j=1,2, \cdots\right\}$ such that (a) $\bar{V}_{k}^{j} \cap$ $\bar{V}_{h}^{i}=\phi$ for $(j, k) \neq(i, h)$; (b) $x^{i_{j}} \in V_{1}^{j} \times \cdots \times V_{n}^{j}$; and (c) $V_{1}^{j} \times \cdots$ $\times V_{n}^{j} \subset U_{i_{j}}$.

Proof. Let $B=\left\{x^{i}\right\}_{i=1}^{\infty}$ satisfy the hypotheses of the lemma. Let $O_{1}^{1}$ be an open set containing $x_{1}^{1}$ such that there is an infinite number of $i$ 's such that $\pi_{k}\left(x^{i}\right) \notin \bar{O}_{1}^{1}$ for $k=1, \cdots, n$ and $\bar{O}_{1}^{1}$ does not contain $x_{j}^{1}$ for $j=2, \cdots, n$. Such an $O_{1}^{1}$ exists by (2) and (3) of the hypotheses of the lemma. Let $\mathrm{B}_{1}=\left\{x^{i j}\right\}_{j=1}^{\infty}$ be the set of all $x^{i}$ 's such that $\pi_{k}\left(x^{i}\right) \notin \bar{O}_{1}^{1}$ for $k=1, \cdots, n$ or $i=1$. Then let $O_{2}^{1}$ be an open set containing $x_{2}^{1}$ such that for an infinite subset of $B_{1}, \pi_{k}\left(x^{i}\right) \notin \bar{O}_{2}^{1}$ for $k=1, \cdots, n$; $\bar{O}_{2}^{1}$ does not contain $x_{j}^{1}$ for $j \neq 2$; and $O_{2}^{1} \cap O_{1}^{1}=\phi$. Such an $O_{2}^{1}$ exists by (2) and (3) of the lemma. Let $B_{2}=\left\{x^{i}: \pi_{k}\left(x^{i}\right) \notin \bar{O}_{\jmath}^{1}\right.$ for $k=1, \cdots, n$ and $j=1$ and 2 or $i=1$ \}. Continuing this process $n$ times we arrive an $n$ infinite subsequences of $B,\left\{B_{1}, \cdots, B_{n}\right\}$ and open sets in $X$, $\left\{O_{1}^{1}, \cdots, O_{n}^{1}\right\}$, with (1) $O_{i}^{1} \cap O_{j}^{1}=\phi$ for $i \neq j$; (2) $x_{j}^{1} \in O_{j}^{1}$ for $j=1, \cdots, n$; and (3) $B_{j}=\left\{x^{i}: \pi_{k}\left(x^{i}\right) \notin \bar{O}_{q}^{1}\right.$ for $k=1, \cdots, n$ and $\left.q=1, \cdots, j\right\} \cup\left\{x^{1}\right\}$. Now let $\left\{V_{1}^{1}, \cdots, V_{n}^{1}\right\}$ be open subsets of $X$ with the property that $x_{j}^{1} \in V_{j}^{1} \subset \bar{V}_{j}^{1} \subset O_{j}^{1}$ and $V_{1}^{1} \times \cdots \times V_{n}^{1} \subset U_{1}$.

Now let $C_{1}=B_{n}-\left\{x^{1}\right\}$ and $X_{1}=X-\bigcup_{i=1}^{n} O_{i}^{1}$. Then $C_{1} \subset\left(\text { int }_{X} X_{1}\right)^{n}$ and $C_{1}$ together with $X_{1}$ satisfies the three hypotheses of the lemma. Let $x^{i_{2}}$ be the first element of $C_{1}$. Then repeating the construction described above we can get open sets in $X_{1}$ which we can also suppose are open in $X,\left\{O_{1}^{2}, \cdots, O_{n}^{2}\right\}$ and $\left\{V_{1}^{2}, \cdots, V_{n}^{2}\right\}$, and an infinite subsequence $C_{2}$ of $C_{1}$ such that (1) $x_{j}^{i_{2}} \in V_{j}^{2} \subset \bar{V}_{j}^{2} \subset O_{j}^{2}$ for all $j$; (2) $V_{1}^{2} \times \cdots$ $\times V_{n}^{2} \subset U_{i_{2}}$; and (3) $C_{2} \subset\left(\text { int }_{X} X_{2}\right)^{n}$ where $X_{2}=X_{1}-\bigcup_{i=1}^{n} O_{i}^{2}$. Let $x^{i_{3}}$ be the first element of $C_{2}$. Continuing this process inductively we get a subsequence $B^{\prime}=\left\{x^{i}\right\}_{j=1}^{\infty}$ and open sets $\left\{V_{k}^{j}: k=1, \cdots, n ; j=1,2, \cdots\right\}$ satisfying the conclusion of the lemma.

Proof of Proposition 1.1. By induction on $n$. If $n=1$, the proposition is clearly true. Suppose $n>1$ and consider the following cases.

Case (i). $X^{n-1}$ is not pseudocompact.

In this case we apply our induction hypothesis to get sets $\left\{U_{1}^{i} \times\right.$ $\left.\cdots \times U_{n-1}^{i}\right\}_{i=1}^{\infty}$ satisfying the conclusion of Proposition 1.1 for $X^{n-1}$. Then define $\left\{V_{j}^{i}: j=1, \cdots, n ; i=1,2, \cdots\right\}$ such that $V_{j}^{i}=U_{j}^{2 i}$ for $j=1, \cdots, n-1$ and $V_{n}^{i}=U_{1}^{2 i+1}$. Then $\left\{V_{\jmath}^{i}\right\}$ can be easily seen to satisfy the conclusion of Porposition 1.1. 
Case (ii). $X^{n-1}$ is pseudocompact.

In this case let $B=\left\{x^{i}\right\}_{i=1}^{\infty}$ be a countably infinite $C$-imbedded subset of $X^{n}$ with $x^{i} \in U_{i}$ an open set in $X^{n}$ with $\left\{U_{i}\right\}_{i=1}^{\infty}$ a discrete collection in $X^{n}$. We claim that $B$ satisfies the conditions of Lemma 1.2. Suppose that for some $i$ and some subsequence $B^{\prime}$ of $B, C 1_{X} \pi_{i}\left[B^{\prime}\right]$ is compact. Then $\mathrm{C} 1_{X} \pi_{i}\left[B^{\prime}\right] \times X^{n-1}$ is pseudocompact [2, E 3.9. E, p. 151]. But $B^{\prime} \subset \mathrm{C1}_{X} \pi_{i}\left[B^{\prime}\right] \times X^{n-1}$ is $C$-imbedded in $X^{n}$, hence in $\mathrm{C} 1_{X} \pi_{i}\left[B^{\prime}\right] \times X^{n-1}$, a contradiction. Thus conditions (1) and (2) of 1.2 are satisfied. If we let $X_{i j}=\left\{\left(x_{1}, \cdots, x_{n}\right) \in X^{n}: x_{i}=x_{j}\right\}$ and $A=\bigcup_{i \neq j} X_{i j}$, then noticing that there are only a finite number of the $X_{i j}$ 's and that each $X_{i j}$ is homeomorphic to $X^{n-1}$ we get that $U_{i} \cap A=\phi$ except for a finite number of $i$ 's or $X^{n-1}$ would not be pseudocompact. By eliminating that finite number of $i$ 's we may assume $B \subset X^{n}-A$ and thus that $B$ satisfies condition (3) of 1.2. Now let $\left\{V_{k}^{i}: k=1\right.$, $\cdots, n ; j=1,2, \cdots\}$ and $B^{\prime}=\left\{x^{i j}\right\}_{j=1}^{\infty}$ satisfy the conclusion of Lemma 1.2. Then $O_{i}=V_{1}^{i} \times \cdots \times V_{n}^{i}$ satisfies the conclusion of Proposition 1.1.

TheOREM 1.3. For all $n, \mathscr{F}_{n}(X)$ is pseudocompact if and only if $X^{n}$ is pseudocompact.

Proof. Let $p: X^{n} \rightarrow \mathscr{F}_{n}(X)$ be defined by

$$
p\left(\left(x_{1}, \cdots, x_{n}\right)\right)=\left\{x_{1}, \cdots, x_{n}\right\} .
$$

Then $p$ is continuous and closed. Also $p \mid\left(X^{n}-A\right)$ is a local homeomorphism, hence open onto $\mathscr{F}_{n}(X)-\mathscr{F}_{n-1}(X)$, where $A$ is defined as in the previous proof (see [4]). If $X^{n}$ is pseudocompact, then $\mathscr{F}_{n}(X)$ is since pseudocompactness is preserved under continuous transformation. Now suppose that $X^{n}$ is not pseudocompact. Let $\left\{U_{k}^{i}: k=\right.$ $1, \cdots, n ; i=1,2, \cdots\}$ satisfy the conclusions of Proposition 1.1. Let $O_{i}=U_{1}^{i} \times \cdots \times U_{n}^{i}$. Then $\left\{p\left(O_{i}\right)\right\}_{i=1}^{\infty}$ can be seen to be a discrete collection of nonempty open sets in $\mathscr{F}_{n}(X)$. Thus $\mathscr{F}_{n}(X)$ is not pseudocompact.

TheOREM 1.4. Let $n \geqq 2$. Then $\beta \mathscr{F}_{n}(X)=\mathscr{F}_{n}(\beta X)$ if and only if $\mathscr{F}_{n}(X)$ is pseudocompact.

Proof. Note that for $n=1, \beta \mathscr{F}_{1}(X)=\mathscr{F}_{1}(\beta X)$ with no assumptions. Suppose that $\mathscr{F}_{n}(X)$ is pseudocompact. Then by Theorem 1.3, $X^{n}$ is also pseudocompact. Thus by [7, Th. 1, p. 371], $\beta\left(X^{n}\right)=(\beta X)^{n}$. Now let $f: \mathscr{F}_{n}(X) \rightarrow[0,1]$ be continuous and let $F: X^{n} \rightarrow[0,1]$ be defined by $F=f \circ p$ where $p: X^{n} \rightarrow \mathscr{F}_{n}(X)$ is as defined in the proof of Theorem 1.3. Now $F$ has an extension $F^{*}:(\beta X)^{n} \rightarrow[0,1]$ since $\beta\left(X^{n}\right)=$ $(\beta X)^{n}$. Consider the map $p^{*}:(\beta X)^{n} \rightarrow \mathscr{F}_{n}(\beta X)$ defined by

$$
p^{*}\left(\left(x_{1}, \cdots, x_{n}\right)\right)=\left\{x_{1}, \cdots, x_{n}\right\} .
$$


Clearly $p^{*}$ is an extension of $p$ and a quotient map [4]. If we can show that $F^{*}$ is constant on the point inverses of $p^{*}$, then by defining $f^{*}$ by $F^{*} \circ p^{*^{-1}}, f^{*}$ will be well defined. Also $f^{*}$ will be continuous by [1, Th. 3.2, p. 123] and an extension of $f$ to $\mathscr{F}_{n}^{2}(\beta X)$. Thus

$$
\mathscr{F}_{n}(\beta X)=\beta \mathscr{F}_{n}(X)
$$

by [6, Th. 6.5, p. 86]. Thus it will be sufficient to show that $F^{*}$ is constant on the point inverses of $p^{*}$. To that end let $\left\{x_{1}, \cdots, x_{k}\right\} \in$ $\mathscr{F}_{n}(\beta X)$ with $x_{i} \neq x_{j}$ for $i \neq j$. Let

$$
p^{*}\left(\left(z_{1}, \cdots, z_{n}\right)\right)=p^{*}\left(\left(y_{1}, \cdots, y_{n}\right)\right)=\left\{x_{1}, \cdots, x_{k}\right\} .
$$

One can construct a net $\left\{x_{1}^{\alpha}, \cdots, x_{k}^{\alpha}\right\}$ of elements $x_{i}^{\alpha}$ in $X$ converging to $\left\{x_{1}, \cdots, x_{k}\right\}$ in $\mathscr{F}_{n}(\beta X)$ such that $x_{i}^{\alpha} \neq{ }_{j}^{\alpha}$ for $i \neq j$ for each $\alpha$ and $x_{i}^{\alpha} \rightarrow x_{i}$ for all $i$. Now if $z_{i}=x_{j}$ let $z_{i}^{\alpha}=x_{j}^{\alpha}$, and if $y_{i}=x_{j}$ let $y_{i}^{\alpha}=$ $x_{\jmath}^{\alpha}$, for all $\alpha$. Then $\left(y_{1}^{\alpha}, \cdots, y_{n}^{\alpha}\right) \rightarrow\left(y_{1}, \cdots, y_{n}\right)$ in $X^{n}$ and $\left(z_{1}^{\alpha}, \cdots, z_{n}^{\alpha}\right) \rightarrow$ $\left(z_{1}, \cdots, z_{n}\right)$ in $X^{n}$. Thus $F^{*}\left(\left(y_{1}^{\alpha}, \cdots, y_{n}^{\alpha}\right)\right) \rightarrow F^{*}\left(\left(y_{1}, \cdots, y_{n}\right)\right)$ and

$$
F^{*}\left(\left(z_{1}^{\alpha}, \cdots, z_{n}^{\alpha}\right)\right) \rightarrow F^{*}\left(\left(z_{1}, \cdots z_{n}\right)\right) \text {. }
$$

Since $F^{*}\left(\left(z_{1}^{\alpha}, \cdots z_{n}^{\alpha}\right)\right)=F^{*}\left(\left(y_{1}^{\alpha}, \cdots, y_{n}^{\alpha}\right)\right)$ for each $\alpha$, this implies

$$
F^{*}\left(\left(y_{1}, \cdots, y_{n}\right)\right)=F^{*}\left(\left(z_{1}, \cdots z_{n}\right)\right) .
$$

Thus $F^{*}$ is constant on the point inverses of $p^{*}$ and the first half of the theorem is proved.

For the converse we will draw upon Proposition 1.1. Suppose that $\mathscr{F}_{n}(X)$ is not pseudocompact. Then $X^{n}$ is not pseudocompact. Let $\left\{U_{k}^{i}: k=1, \cdots n ; i=1,2, \cdots\right\}$ be as in Proposition 1.1. Let

$$
\mathscr{U}_{i}=\left\langle U_{1}^{i}, \cdots, U_{n}^{i}\right\rangle \cap \mathscr{F}_{n}(X)=p\left[U_{1}^{i} \times \cdots \times U_{n}^{i}\right]
$$

in $\mathscr{F}_{n}(X)$ (see [13] for notation). Then one can show that $\left\{\mathscr{U}_{i}\right\}_{i=1}^{\infty}$ is a discrete collection of open sets in $\mathscr{F}_{n}(X)$. Let $B_{i} \in \mathscr{U}_{i}$ and $f: \mathscr{F}_{n}(X) \rightarrow$ $[0,1]$ be defined so that $f\left(B_{i}\right)=1$ and $f(B)=0$ for all $B \notin \bigcup_{i=1}^{\infty} \mathscr{Q}_{i}$. Now if $\mathscr{F}_{n}(\beta X)$ were equivalent to $\beta \mathscr{F}_{n}(X)$, there would be a continuous extension of $f$ to some $f^{*}: \mathscr{F}_{n}(\beta X) \rightarrow[0,1]$. We will show that no extension of $f$ to $\mathscr{F}_{n}(\beta X)$ is continuous. Let $B_{0}$ be a limit point of $\left\{B_{i}\right\}_{i=1}^{\infty}$ in $\mathscr{F}_{n}(\beta X)$. Let $\mathscr{U}=\left\langle U_{1}, \cdots, U_{n}\right\rangle \cap \mathscr{F}_{n}(\beta X)$ be a neighborhood of $B_{0}$ in $\mathscr{F}_{n}(\beta X)$. Let $B_{i_{1}}$ and $B_{i_{2}}$ be distinct with $B_{i_{1}}$ and $B_{i_{2}}$ in $\mathscr{U}$. Let $B$ be defined by $B=\left\{p_{1}, \cdots, p_{n}\right\}$ where $p_{j} \in B_{i_{1}} \cap$ $U_{j}$ for $j$ odd and $p_{j} \in B_{i_{2}} \cap U_{j}$ for $j$ even. Then $B \in \mathscr{U}$. But also $B \notin \bigcup_{i=1}^{\infty} \mathscr{U}_{i}$. Thus $f^{*}(B)=f(B)=0$. Thus in every neighborhood $\mathscr{U}$ of $B_{0}$ in $\mathscr{F}_{n}(\beta X), f^{*}$ takes on the value 0 and the value 1 , a contradiction. Thus $\mathscr{F}_{n}(\beta X) \neq \beta \mathscr{F}_{n}(X)$.

2. Results without the aid of $\mathrm{CH}$. In [9] it is shown that if 
$X$ is a well ordered space with the order topology, then $2^{X}$ normal implies $X$ compact. In [10] it is shown that $2^{2^{X}}$ is normal if and only if $X$ is compact. In this section we use Theorem 1.4 to show that for certain classes of spaces $X, 2^{X}$ is not normal. The most positive result in this paper assumes $\mathrm{CH}$ and will be proved in the next section making use of the results of this section and [10].

Lemma 2.1. If $\left\{F_{i}\right\}_{i=1}^{\infty}$ is a countable collection of closed sets in a normal countably compact space $X$, then

$$
\mathrm{C1}_{\beta X}\left[\bigcap_{i=1}^{\infty} F_{i}\right]=\bigcap_{i=1}^{\infty} \mathrm{C1}_{\beta X} F_{i} .
$$

Proof. Clearly $\mathrm{C1}_{\beta X}\left[\bigcap_{i=1}^{\infty} F_{i}\right] \subset \bigcap_{i=1}^{\infty} \mathrm{C1}_{\beta X} F_{i}$. Now suppose the contrary and let $x \in \mathrm{C1}_{\beta X} F_{i}$ for all $i$ with $x \notin \mathrm{C} 1_{\beta X}\left[\bigcap_{i=1}^{\infty} F_{i}\right]$. Then let $V$ be an open set in $\beta X$ containing $x$ such that $\mathrm{C1}_{\beta X} V \cap \mathrm{C1}_{\beta X}\left[\bigcap_{i=1}^{\infty} F_{i}\right]=$ $\phi$. Let $U=V \cap X$ and note that $\mathrm{C1}_{\beta X} V=\mathrm{C1}_{\beta_{X}} U$. Clearly $\left(\mathrm{C1}_{X} U\right) \cap$ $\left[\bigcap_{i=1}^{\infty} F_{i}\right]=\phi . \quad$ By the countable compactness of $X$ there is an $n$ such

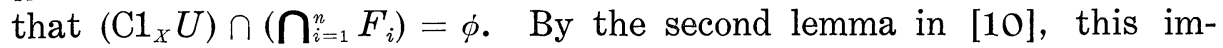
plies that $\left(\mathrm{C1}_{\beta X} U\right) \cap\left[\bigcap_{i=1}^{n} \mathrm{C}_{\beta_{X}} F_{i}\right]=\phi$. However, $x \in \mathrm{C}_{\beta_{X}} U$ and $x \in$ $\mathrm{C}_{\beta X} F_{i}$ for $i=1, \cdots, n$, a contradiction. Thus

$$
\mathrm{C1}_{\beta X}\left[\bigcap_{i=1}^{\infty} F_{i}\right]=\bigcap_{i=1}^{\infty} \mathrm{Cl}_{\beta X} F_{i}
$$

as asserted.

Proposition. If $2^{X}$ is normal, then $X$ is normal and countably compact. If, in addition, $X$ is not compact, then there is an $n$ such that $X^{n}$ is not pseudocompact.

Proof. Suppose that $2^{X}$ is normal. Then $X$ is normal and countably compact [10, corollary to Th. 1]. Suppose that $X$ is not compact and that $X^{n}$ is pseudocompact for all $n$. Let $x \in \beta X-X$ and let $\mathscr{F}_{x}=\left\{F: F\right.$ is closed in $X$ and $\mathrm{C1}_{\beta X} F$ contains $\left.x\right\}$. Let $\hat{X}$ be the set of singletons $\{\{x\}: x \in X\}$. Then $\mathscr{F}_{x}$ and $\hat{X}$ are closed subsets of $2^{X}$ and disjoint. We will show that $\mathscr{F}_{x}$ and $\hat{X}$ cannot be separated by a continuous real valued function. Suppose that $f: 2^{X} \rightarrow[0,1]$ is continuous with $f \mid X \equiv 0$. Let $f_{n}$ be the restriction of $f$ to $\mathscr{F}_{n}(X)$ for each $n$. By Theorem $1.3 \mathscr{F}_{n}(X)$ is pseudocompact. Thus by Theorem 1.4, $f_{n}$ has an extension $f_{n}^{*}$ to $\mathscr{F}_{n}(\beta X)$ for each $n$. Clearly $f_{n}^{*}(x)=0$ for all $n$. For each $n$, let $U_{n}$ be a neighborhood of $x$ in $\beta X$ such that for $A \in 2^{U} \cap \cap \mathscr{F}_{n}(\beta X)$ we have $f_{n}^{*}(A) \leqq 2^{-n}$. Let

$$
F_{n}=\mathrm{C} 1_{X}\left(U_{n} \cap X\right) \text {. }
$$

Then $x \in \mathrm{C}_{\beta_{X}} F_{n}$ for each $n$. Thus $x \in \mathrm{C1}_{\beta X}\left[\bigcap_{i=1}^{\infty} F_{i}\right]$ by Lemma 2.1. Thus $\bigcap_{i=1}^{\infty} F_{i}=F_{0}$ is an element of $\mathscr{F}_{x}$. Let $B$ be any finite subset 
of $F_{0}$. Then if card $B=k$, then $B \in 2^{U_{n}} \cap \mathscr{F}_{n}(\beta X)$ for all $n \geqq k$. Thus $f_{n}(B)=f(B) \leqq 2^{-n}$ for all $n \geqq k$. Thus $f(B)=0$. This implies that $f\left(F_{0}\right)=0$. Therefore $\hat{X}$ and $\mathscr{F}_{x}$ cannot be separated, a contradiction. Thus $X^{n}$ must be nonpseudocompact for some $n$.

REMARK 2.3. It is not known if $X$ normal and countably compact implies $X^{n}$ pseudocompact for all $n$. All of the examples known to the author, for example Frolík's [3], of a completely regular space $X$ which is countably compact and such that $X^{n}$ is not pseudocompact are obtained by choosing an appropriate dense subset $A$ of

$$
N^{*}=\beta N-N
$$

and letting $X=N \cup A$. Assuming $\mathrm{CH}$, all such examples are nonnormal by the result of Gillman and Fine [5] that proper dense subsets of $N^{*}$ are not $C^{*}$-imbedded in $N^{*}$. If the normality and countable compactness of $X$ implies $X^{n}$ pseudocompact for all $n$, then $2^{X}$ normal implies $X$ compact without assuming $\mathrm{CH}$. However, this would be an interesting result even if $\mathrm{CH}$ were required in proving it.

Proposition 2.4. If $X$ is a countably compact $k$-space and $Y$ is countably compact, then $X \times Y$ is countably compact. Thus $X^{n}$ is countably compact for all $n$.

Proof. Proof of the first part of the proposition can be found in [14, Th. 1.1]. The second part follows by induction.

DEFINITION 2.5. A space is strongly countably compact if the closure of every countable set is compact [10]. A space is sequent $i$ ally compact if each sequence has a convergent subsequence.

CoRollary 2.6. If $X$ has any of the following properties, then $2^{X}$ normal implies $X$ compact.

(a) $X$ first countable,

(b) X locally compact,

(c) X a k-space,

(d) X strongly countably compact, and

(e) $X$ sequentially compact.

Proof. For the definition of a $k$-space see [1, Definition 9.2, p. 248]. By [1, 9.3, p. 248] (c) implies (a) and (b). But (c) follows from Proposition 2.2 and Proposition 2.4.

For (d) and (e), one can show that these properties are finitely productive. Thus in these cases $X^{n}$ is pseudocompact for all $n$ and 
Proposition 2.2 can be applied.

We conclude this section with a minor result.

LEMMA 2.7. If $X$ is separable and countably compact, then $X \times \beta N$ normal implies $X$ compact.

Proof. Let $f: \beta N \rightarrow \beta X$ be continuous and surjective. If $X \times$ $\beta N$ is normal, then so is $X \times \beta X$ since the map $g=i \times f: X \times \beta N \rightarrow$ $X \times \beta X$ is closed. But $X \times \beta X$ is normal if and only if $X$ is paracompact [16, Th. 2, p. 1046]. But paracompactness and countable compactness imply compactness [1, Corollary 3.4, p. 230]. Thus $X$ is compact.

Theorem 2.8. If $2^{X} \times \beta N$ is normal, then $X$ is compact.

Proof. Let $\hat{X}=\{\{x\}: x \in X\}$. Then $\hat{X}$ is a homeomorphic copy of $X$ [12, Corollary 3a, p. 166] and closed in $2^{X}$ [13, Proposition 2.4.2, p. 156]. Let $K$ be the closure of any countable subset of $X$. Then $K$ is countably compact. Now $\hat{K} \times \beta N$ is a closed subset of $2^{X} \times \beta N$, hence normal. Thus $K$ is compact by Lemma 2.6. Thus $X$ is strongly countably compact. But $2^{X}$ is normal since $2^{X} \times \beta N$ is, and thus $X$ is compact by Corollary 2.6(d).

3. Results assuming $\mathrm{CH}$. In [10, proof of Th. 4] it is shown that if $X$ is not compact, then there is an initial ordinal $\omega_{\alpha}$ such that $\left[0, \omega_{\alpha}\right)$ can be imbedded as a closed subset of $2^{x}$. If we let the imbedding be $f(\beta)=F_{\beta}$, then the set $\left\{F_{\beta}: \beta<\omega_{\alpha}\right\}$ has the property that (1) for $\gamma>\beta, F_{\gamma} \varsubsetneqq F_{\beta}$; (2) if $\gamma$ is a limit ordinal $F_{\gamma}=\cap\left\{F_{\beta}: \beta<\gamma\right\}$; and (3) $\cap\left\{F_{\beta}: \beta<\omega_{\alpha}\right\}=\phi$. This result will form an important part of what follows.

Recall that a regular open set $V$ is one which has the property that $V=\operatorname{int} \bar{V}$. If $B$ is a dense subset of $X$ and $V$ is a regular open set in $X$, then $U=V \cap B$ is a regular open set in $B$.

Lemma 3.1. If $A$ is a discrete subset of $X$ with $X$ separable, then card $A \leqq 2^{\omega_{0}}$.

Proof. For each $x \in A$ let $V_{x}$ be a regular open set in $X$ such that $V_{x} \cap A=\{x\}$. Let $U_{x}=V_{x} \cap B$ where $B$ is a countable dense set in $X$. Then for $x \neq y, U_{x} \neq U_{y}$. Thus the map $g(x)=U_{X}$ is one to one into the power set of $B$. Thus card $A \leqq 2^{\omega_{0}}$.

Proposition 3.2. Assume CH. Suppose that $X$ is separable and 
countably compact but not compact. Then $\left[0, \omega_{1}\right)$ can be imbedded in $2^{X}$ as a closed subset.

Proof. We make use of the results in [10] described above to say that $\left[0, \omega_{\alpha}\right)$ can be imbedded in $2^{X}$ for some initial ordinal $\omega_{\alpha}$. Since $X$ is separable, so is $2^{X}$. Let $A$ be the nonlimit points of $\left[0, \omega_{\alpha}\right.$ ). Then card $A=\omega_{\alpha}$ and $A$ is discrete. Thus $\omega_{\alpha} \leqq 2^{\omega_{0}}$ by Lemma 3.1. Assuming $\mathrm{CH} \omega_{\alpha}=\omega_{0}$ or $\omega_{\alpha}=\omega_{1}$. If $\omega_{\alpha}=\omega_{0}$, then by (3) above, $X$ would not be countably compact. Thus $\omega_{\alpha}=\omega_{1}$ and $\left[0, \omega_{1}\right)$ is a closed subset of $2^{X}$.

Proposition 3.3. Assume CH. Suppose that $X$ is separable, countably compact, and not first countable. Then $\left[0, \omega_{1}\right]$ can be imbedded in $2^{x}$.

Proof. Let $\left\{V_{\alpha}\right\}$ be a neighborhood basis for $x$ in $X$ where $X$ is not first countable at $x$. Since $X$ is separable we may assume that $\left\{V_{\alpha}\right\}$ has cardinality $\omega_{\alpha} \leqq 2^{\omega_{0}}$. Since $X$ is not first countable at $x$, $\omega_{\alpha}>\omega_{0}$. Thus card $\left\{V_{\alpha}\right\}=\omega_{1}$ and we may assume that the $V_{\alpha}$ 's are indexed by the countable ordinals. We now define closed sets $\left\{F_{\beta}\right.$ : $\left.\beta<\omega_{1}\right\}$ having the following properties: (1) $F_{\beta+1} \subset \bar{V}_{\beta}$ for all $\beta$; (2) $\gamma>\beta$ implies that $F_{\gamma} \varsubsetneqq F_{\beta}$; (3) if $\gamma$ is a limit ordinal, then

$$
F_{\gamma}=\cap\left\{F_{\beta}: \beta<\gamma\right\} ;
$$

and (4) $\cap\left\{F_{\beta}: \beta<\omega_{1}\right\}=\{x\}$. The construction is as follows: let $\alpha_{0}=1$. Having defined a subsequence of the countable ordinals $\left\{\alpha_{\beta}: \beta<\gamma\right\}$ let $\alpha_{\gamma}=\sup \left\{\alpha_{\beta}: \beta<\gamma\right\}$ if $\gamma$ is a limit ordinal. Otherwise let $\alpha_{r}$ be the first $\alpha$ such that if $F=\cap\left\{\bar{V}_{\lambda}: \lambda<\alpha_{\beta}\right.$ some $\left.\beta<\gamma\right\}$, then $F-\bar{V}_{\alpha} \neq \phi$. Note that by the countable compactness of $X$ and the fact that $X$ is not first countable at $x, F \neq\{x\}$ and thus such an $\alpha_{r}$ exists. Continue the process inductively and let $\left\{\alpha_{\beta}: \beta<\omega_{1}\right\}$ be the sequence so defined. Then let $F_{\beta}=\cap\left\{\bar{V}_{\alpha}: \alpha<\alpha_{\beta}\right\}$. Then $\left\{F_{\beta}: \beta<\omega_{1}\right\}$ satisfies (1), (2), (3), and (4) above. Let us define $F_{\omega_{1}}=\{x\}$. Then we claim that $\left\{F_{\beta}: \beta \leqq \omega_{1}\right\}$ is our desired set.

Claim. The map $f(\beta)=F_{\beta}$ is a homeomorphism of $\left[0, \omega_{1}\right]$ into $2^{x}$.

Proof of claim: Clearly $f:\left[0, \omega_{1}\right] \rightarrow\left\{F_{\beta}\right\}$ is one to one and onto. Suppose that $\alpha$ is a countable limit ordinal. Then $F_{\alpha}=\cap\left\{F_{\beta}: \beta<\alpha\right\}$ by (3) above. Let $F_{\alpha} \in\left\langle U_{1}, \cdots, U_{n}\right\rangle$. We may suppose that $\left\langle U_{1}, \cdots, U_{n}\right\rangle \cap\left\{F_{\beta}\right\}=\left\{F_{\beta}: \beta \leqq \alpha \quad\right.$ and $\left.\quad F_{\beta} \subset \bigcup_{i=1}^{n} U_{i}\right\}$ by supposing some $U_{i}=X-F_{\alpha+1}$. Suppose that $\beta_{i} \rightarrow \alpha$ with $F_{\beta_{i}} \not \subset \bigcup_{i=1}^{n} U_{i}$. Then letting $G_{i}=F_{\beta_{i}}-\bigcup_{j=1}^{n} U_{j},\left\{G_{i}\right\}_{i=1}^{\infty}$ has the finite intersection property 
and empty intersection, contradicting the countable compactness of $X$. Thus there is no such sequence $\beta_{i}$ converging to $\alpha$ and $f$ is continuous at $\alpha$. Now consider $\omega_{1}$. Let $U$ be any open set in $X$ containing $\{x\}$. Let $\alpha$ be such that $\bar{V}_{\alpha} \subset U$. Then for all $\beta>\alpha$, $F_{\beta} \in 2^{U}$. Thus $f$ is continuous at $\omega_{1}$. Thus $f$ is homeomorphism onto $\left\{F_{\beta}: \beta \leqq \omega_{1}\right\}$.

THEOREM 3.4. Assume CH. Then $2^{X}$ is normal if and only if $X$ is compact.

Proof. We need only show that if $2^{X}$ is normal, then $X$ is compact. Assume that $2^{X}$ is normal. Let $K$ be the closure of any countable subset of $X$. Then $2^{K}$ is also hormal since it is a closed subspace of $2^{X}$. If we can show that for any separable space $Z, 2^{Z}$ normal implies that $Z$ is compact, then $K$ will have to be compact. Thus $X$ will be strongly countably compact and compact by Corollary $2.6(d)$.

ClaIM. If $Z$ is separable and $2^{Z}$ is normal, then $Z$ is compact.

Proof of claim. Suppose that $Z$ is separable and not compact with $2^{Z}$ normal. By Corollary 2.6(a) $Z$ is not first countable. Suppose that $Z$ is not first countable at the point $x$. Let $O$ be an open set containing $x$ such that $Z-O$ is not compact. Such an $O$ exists since $X$ is not compact. Let $P$ be an open set containing $x$ such that $\bar{P} \subset O$. Let $U=Z-\mathrm{C} 1(Z-\bar{P})$. Then $U$ has the property that $Z-U$ is separable and not compact. Now let $V$ be an open set containing $x$ with $\bar{V} \subset U$. Let $K_{1}=\bar{V}$ and $K_{2}=X-U$. Then let $K=$ $K_{1} \cup K_{2}$. Now $K$ is a closed subset of $Z$ and $2^{K}$ is a closed subspace of $2^{Z}$ as remarked in the preliminaries. Also $2^{K}$ is homeomorphic to $2^{K_{1}} \times 2^{K_{2}}$. But by Proposition $3.2\left[0, \omega_{1}\right.$ ) can be imbedded as a closed subset of $2^{K_{2}}$. By Proposition 3.3 we can imbed [0, $\left.\omega_{1}\right]$ as a closed subset of $2^{K_{1}}$. Thus $\left[0, \omega_{1}\right] \times\left[0, \omega_{1}\right)$ is a closed subset of $2^{K}$ and thus of $2^{Z}$. But $\left[0, \omega_{1}\right] \times\left[0, \omega_{1}\right)$ is not normal by [16, Th. 2, p. 1046] or [5, 8M(4), p. 129]. This implies that $2^{Z}$ is not normal, a contradiction. Thus $Z$ must be compact.

This proves the claim and completes the proof of Theorem 3.4.

THEOREM 3.5. Assume CH. The following are equivalent.
(a) $X$ is compact,
(b) $2^{X}$ is compact,
(c) $2^{X}$ is normal,
(d) $2^{X}$ is meta-Lindelöf, and
(e) $2^{2^{x}}$ is regular. 
Proof. The equivalence of (a), (b), and (d) is shown in [10] without $\mathrm{CH}$. The equivalence of (c) and (e) is given in [13, Th. 4.9, p. 163]. By Theorem 3.4 (a) and (c) are equivalent.

REMARK 3.6. It is trivial to see that the assumption that $X$ is completely regular can be reduced to $X$ being Hausdorff in Theorem 3.4 , since $2^{X}$ normal will then imply that $X$ is completely regular since it is a subspace of $2^{X}$. It would have been a nuisance to keep stating different hypotheses for $X$ for each new theorem, but many can be trivially reduced as in this case.

\section{REFERENCES}

1. J. Dugundji, Topology, Allyn and Bacon, Boston, 1966.

2. R. Engelking, Outline of general topology, John Wiley, 1968.

3. Z. Frolík, Sums of ultrafilters, Bull. Amer. Math. Soc. 73 (1967), 87-91.

4. T. Ganea, Symmetrische Potenzen topologischer Räume, Math. Nach. 11 (1954), 305316.

5. L. Gillman, The space $\beta N$ and the continuum hypothesis, General Topology and its Relations to Modern Analysis and Algebra, II (Proc. Second Prague Topological Sympos., 1966), Academia, Prague, 1967, 144-146.

6. L. Gillman and M. Jerison, Rings of continuous functions, Van Nostrand, New York, 1960.

7. I. Glicksberg, Stone-Čech compactifications of products, Trans. Amer. Math. Soc. 90 (1959), 369-382.

8. J. Isbell, Uniform Spaces, Mathematical Surveys No. 12, American Mathematical Society, Providence, 1964.

9. V. M. Ivanova, On the theory of the space of subsets, (in Russian) Dokl. Akad. Nauk SSSR 101 (1955), 601-603.

10. J. Keesling. Normality and properties related to compactness in hyperspaces, Proc. Amer. Math. Soc. 24 (1970), 760-766.

11. - Normality and compactness are equivalent in hyperspaces, Bull. Amer. Math. Soc. (to appear)

12. K. Kuratowski, Topology I, Academic Press, New York, 1966.

13. E. Michael, Topologies on spaces of subsets, Trans. Amer. Math. Soc. 71 (1951), 152-182.

14. N. Noble, Countably compact and pseudocompact products, Czech. Math. J. (94) 19 (1969), 390-397.

15. , Products with closed projections, II, (to appear)

16. H. Tamamo, On paracompactness, Pacific J, Math. 10 (1960), 1043-1047.

Received September 26, 1969, and in revised form December 5, 1969.

UNIVERSITY OF FLORIDA

Gainesville, Florida 



\section{PACIFIC JOURNAL OF MATHEMATICS}

\section{EDITORS}

\author{
H. SAMELSON \\ Stanford University \\ Stanford, California 94305 \\ Richard Pierce \\ University of Washington \\ Seattle, Washington 98105
}

J. DUGUNDJI

Department of Mathematics

University of Southern California

Los Angeles, California 90007

RICHARD ARENS

University of California

Los Angeles, California 90024

\section{ASSOCIATE EDITORS}
E. F. BECKENBACH
B. H. NEUMaNN
F. WOLE
K. YosHIDA

\section{SUPPORTING INSTITUTIONS}

\author{
UNIVERSITY OF BRITISH COLUMBIA \\ CALIFORNIA INSTITUTE OF TECHNOLOGY \\ UNIVERSITY OF CALIFORNIA \\ MONTANA STATE UNIVERSITY \\ UNIVERSITY OF NEVADA \\ NEW MEXICO STATE UNIVERSITY \\ OREGON STATE UNIVERSITY \\ UNIVERSITY OF OREGON \\ OSAKA UNIVERSITY \\ UNIVERSITY OF SOUTHERN CALIFORNIA
}

\author{
STANFORD UNIVERSITY \\ UNIVERSITY OF TOKYO \\ UNIVERSITY OF UTAH \\ WASHINGTON STATE UNIVERSITY \\ UNIVERSITY OF WASHINGTON

$* * *{ }^{*} *{ }^{*}$
AMERICAN MATHEMATICAL SOCIETY
CHEVRON RESEARCH CORPORATION
TRW SYSTEMS \\ NAVAL WEAPONS CENTER
}

The Supporting Institutions listed above contribute to the cost of publication of this Journal. but they are not owners or publishers and have no responsibility for its content or policies.

Mathematical papers intended for publication in the Pacific Journal of Mathematics should be in typed form or offset-reproduced, (not dittoed), double spaced with large margins. Underline Greek letters in red, German in green, and script in blue. The first paragraph or two must be capable of being used separately as a synopsis of the entire paper. The editorial "we" must not be used in the synopsis, and items of the bibliography should not be cited there unless absolutely necessary, in which case they must be identified by author and Journal, rather than by item number. Manuscripts, in duplicate if possible, may be sent to any one of the four editors. Please classify according to the scheme of Math. Rev. 36, 1539-1546. All other communications to the editors should be addressed to the managing editor, Richard Arens, University of California, Los Angeles, California, 90024.

50 reprints are provided free for each article; additional copies may be obtained at cost in multiples of 50 .

The Pacific Journal of Mathematics is published monthly. Effective with Volume 16 the price per volume (3 numbers) is $\$ 8.00$; single issues, $\$ 3.00$. Special price for current issues to individual faculty members of supporting institutions and to individual members of the American Mathematical Society: $\$ 4.00$ per volume; single issues $\$ 1.50$. Back numbers are available.

Subscriptions, orders for back numbers, and changes of address should be sent to Pacific Journal of Mathematics, 103 Highland Boulevard, Berkeley, California, 94708.

PUBLISHED BY PACIFIC JOURNAL OF MATHEMATICS, A NON-PROFIT CORPORATION

Printed at Kokusai Bunken Insatsusha (Internatıonal Academic Printing Co., Ltd.), 7-17, Fujimi 2-chome, Chiyoda-ku, Tokyo, Japan. 


\section{Pacific Journal of Mathematics}

\section{Vol. 33, No. 3 \\ May, 1970}

Charles A. Akemann, Approximate units and maximal abelian

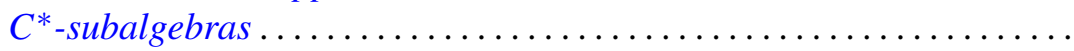

Gail Atneosen, Wild points of cellular arcs in 2-complexes in $E^{3}$ and cellular hulls

John Logan Bryant and De Witt Sumners, On embeddings of 1-dimensional

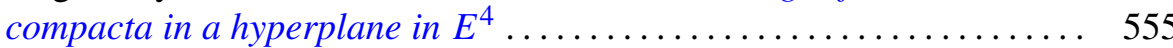

H. P. Dikshit, On a class of Nörlund means and Fourier series.......... 559

Nancy Dykes, Generalizations of realcompact spaces .............. 571

Hector O. Fattorini, Extension and behavior at infinity of solutions of certain linear operational differential equations .................. 583

Neal David Glassman, Cohomology of nonassociative algebras .......... 617

Neal Hart, Ulm's theorem for Abelian groups modulo bounded groups . . . . 635

Don Barker Hinton, Continuous spectra of second-order differential

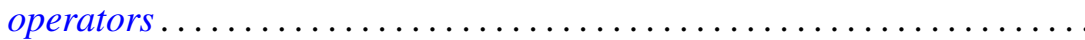

Donald Gordon James, On Witt's theorem for unimodular quadratic forms. II ............................................. 645

Melvin F. Janowitz, Principal multiplicative lattices................ 653

James Edgar Keesling, On the equivalence of normality and compactness in hyperspaces .................................... 657

Adalbert Kerber, Zu einer Arbeit von J. L. Berggren über ambivalente Gruppen

Keizō Kikuchi, Various $m$-representative domains in several complex variables

Jack W. Macki and James Stephen Muldowney, The asymptotic behaviour of solutions to linear systems of ordinary differential equations ....

Andy R. Magid, Locally Galois algebras . . . .

T. S. Ravisankar, On differentiably simple algebras......

Joseph Gail Stampfli, The norm of a derivation ..

Francis C.Y. Tang, On uniqueness of central decompositions of groups ...

749

Robert Charles Thompson, Some matrix factorization theorems. I .

763

Robert Charles Thompson, Some matrix factorization theorems. II . . 